\title{
Criterios para la gestión de personas especialmente vulnerables y trabajadores/as especialmente sensibles frente al SARS-CoV-2 en las empresas de Cataluña en el contexto de pandemia ${ }^{*}$
}

Associació Catalana de Salut Laboral - Grupo de trabajo para la elaboración de criterios para la gestión de personas especialmente vulnerables y trabajadores/ as especialmente sensibles frente al SARS-CoV-2 en las empresas de Cataluña. (GTPSVTESSARS-CoV-2)

08 de abril de $2020^{\text {** }}$

* Este documento, en lo que no diga, asume los criterios oficiales que dicta en cada momento el Departament de Salut de la Generalitat de Catalunya sobre la pandemia del SARS-CoV-2 y la COVID-19. ** Este documento está en revisión permanente en función de la evolución de la pandemia y de la nueva información de que se disponga. Los contenidos son válidos en la fecha de publicación que consta en la portada y que identifica la versión.

Fechas · Dates

Recibido: 2020.04 .09 Aceptado: 2020.04.10 Publicado: 2020.04.15

\section{Correspondencia $\cdot$ Corresponding Author}

Associació Catalana de Salut Laboral

Acadèmia de Ciències Mèdiques i de la Salut de Catalunya i de Balears.

Major de Can Caralleu 1-7, 08017 - Barcelona.

scsl@academia.cat 


\section{Índice}

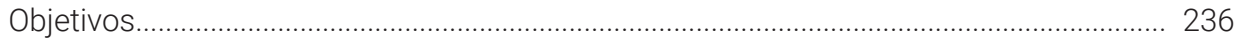

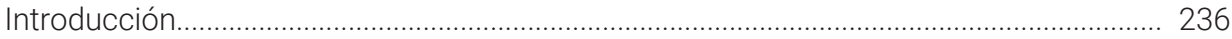

¿Cómo identificar y clasificar el riesgo laboral derivado del SARS-CoV-2? ...................... 238

Tablas orientativas para valorar el riesgo laboral de exposición

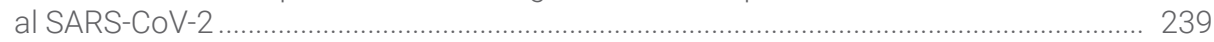

Criterios para orientar la toma de decisiones ............................................................ 239

Concepto de especial vulnerabilidad de una persona y de especial

sensibilidad de un trabajador/a.

Personas especialmente vulnerables (PEV): concepto y terminología.......................... 241

Trabajadores/as especialmente sensibles (TES): concepto y terminología.................. 241

¿Cuáles son los grupos de patologías que pueden incluir personas

especialmente vulnerables (PEV) a la COVID-19?

Listado de grupos de patologías y situaciones para orientar

la determinación de PEV:

Criterios para orientar la toma de decisiones.

¿Qué personas pueden ser consideradas trabajadores/as especialmente sensibles (TES) frente al riesgo laboral derivado del SARS-CoV-2?

Información adicional para situaciones determinadas para ciertas

patologías o tratamientos

Situaciones especiales no vinculadas a enfermedades (aclaraciones): ....................... 247

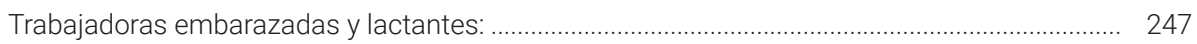

Trabajadores/as de más edad:.............................................................................................. 247

¿Qué actuaciones orientativas hay que llevar a cabo desde el SP ante

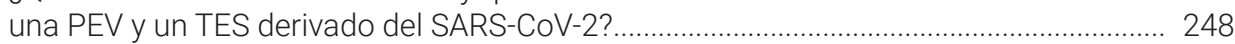

Personas especialmente vulnerables (PEV) ............................................................. 248

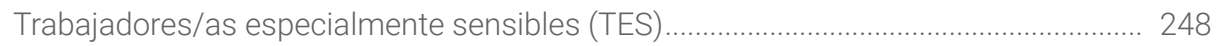

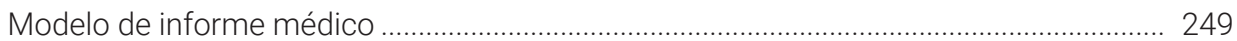

Informe de Vigilancia de la salud por especial sensibilidadal COVID-19 ..................... 249

Siglas y acrónimos utilizados en este documento ............................................................ 250

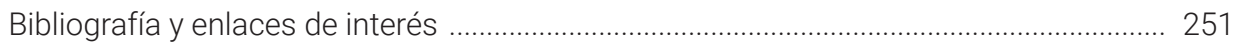

Composición del grupo de trabajo GTPSVTESSARS-CoV-2 y relación

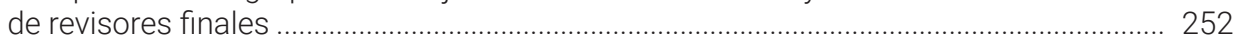

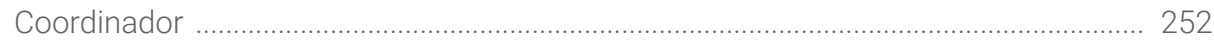

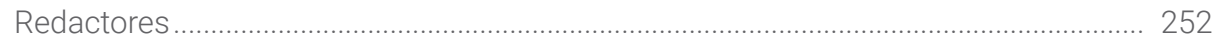

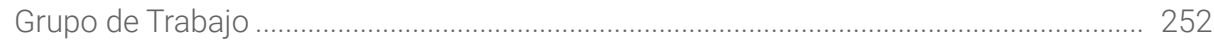

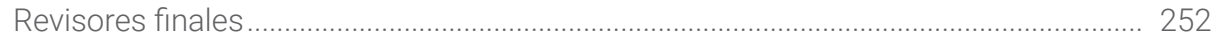




\section{Objetivos}

Elaborar un documento de consenso profesional de Medicina del Trabajo sobre la identificación y gestión, en un entorno de pandemia, de:

- las personas especialmente vulnerables (PEV) frente a la COVID-19 en las empresas,

- los trabajadores/as especialmente sensibles (TES) frente al riesgo laboral de exposición al SARS-CoV-2 en las empresas,

que ayude a tomar las decisiones médicas y laborales, éticas, en torno a las personas y el trabajo que son especialmente complejas en momentos de mucha presión e incertidumbre como los actuales de pandemia por coronavirus SARSCoV-2, y que contribuyan a conciliar los derechos a la salud y al trabajo de los trabajadores/as, a cumplir con sus responsabilidades a las empresas y a garantizar los servicios básicos y esenciales a los ciudadanos.

\section{Introducción}

Los servicios de prevención (SP) deben asesorar y asistir técnicamente a las direcciones de las empresas, a los trabajadores/as y sus representantes, delegados/ as de prevención, sobre los aspectos relativos a la interrelación entre la salud y el trabajo. Hay dos ámbitos sobre los que se concreta especialmente su actividad: por un lado, la identificación y evaluación de riesgos laborales (aquellos riesgos derivados del trabajo) y, por otro, la valoración del estado de salud de las personas valorando la interacción. En ambos casos el resultado es la emisión de dictámenes y recomendaciones que permitan ajustar el binomio salud y trabajo con el máximo beneficio para todos.

En este sentido una de sus tareas relevantes es la identificación de los llamados trabajadores/as especialmente sensibles (TES) con relación a un riesgo determinado a la que, en este momento, se ha añadido la necesidad de hacerlo con relación a la exposición al coronavirus SARS-CoV-2.

Para poder llevarlo a cabo habrá hecho falta identificar y evaluar este riesgo en el trabajo, en las empresas, al tiempo que valorar las condiciones de salud de cada trabajador/a individualmente. Y una vez encaradas las dos informaciones, establecer la naturaleza, en caso de que la haya, de la especial vulnerabilidad y/o de la especial sensibilidad de cada persona frente al riesgo de exposición al SARS-CoV-2.

Esta actividad es la que deben llevar a cabo los profesionales sanitarios de los SP para poder emitir un informe con recomendaciones sobre las medidas específicas adicionales de prevención, adaptación y protección, para conseguir unas condiciones que permitan realizar el trabajo sin que las condiciones psicofísicas de la persona incorporen un riesgo sobreañadido a la posible exposición laboral al coronavirus SARS-CoV-2. 
Es decir, el deber de protección de los TES frente a los riesgos laborales y en concreto al riesgo biológico, y otros riesgos psicosociales añadidos, que ha supuesto la irrupción del SARS-CoV-2 en el entorno laboral, requiere conocer las características personales de los trabajadores/as, su estado biológico y psicológico conocido, o que se pueda conocer, teniendo en cuenta el resultado de la evaluación de los riesgos actualizada, los criterios de especial vulnerabilidad y de especial sensibilidad y, en función de estos, determinar las medidas de prevención y protección que sean necesarias.

Y es que de acuerdo con el artículo 25 de la Ley de Prevención de Riesgos Laborales (LPRL)* ${ }^{*}$ los trabajadores/as no deben ocupar aquellos puestos de trabajo en los que, por razón de sus características personales, estado biológico o vulnerabilidad, puedan, tanto ellos como los demás trabajadores/as y las otras personas relacionadas con la empresa, exponerse a situaciones de riesgo.

Además, en las empresas, hay que considerar la salud de los trabajadores/as de manera holística, especialmente la de aquellas personas especialmente vulnerables a la COVID-19, y en colaboración, desde el sistema de prevención de riesgos laborales, con los sistemas sanitarios, el sistema de salud pública y el de seguridad social, que en muchos momentos confluyen, se complementan o se potencian.

Cada uno de estos sistemas velan por la salud, pero su enfoque y su intención son diversos: preservar la salud de los ciudadanos, proteger la salud colectiva, establecer estrategias de contención de la pandemia, ofrecer apoyo económico a diversas situaciones de vulnerabilidad, como la incapacidad laboral.

Es por ello que en el ámbito individual y en el laboral, son los profesionales sanitarios de los SP quienes deberán dictaminar para proteger la salud de los trabajadores/as en relación con la exposición a los riesgos laborales. Y hacerlo siempre teniendo en cuenta todas las indicaciones y normativas que decreten o reglamenten las instituciones públicas.

Ante ello resulta que, en el ámbito de la medicina del trabajo, es frecuente encontrar comportamientos y decisiones diversas ante problemas o situaciones idénticas o muy similares. Y hay gran variabilidad de resultados incluso en los más sencillos ejercicios de la práctica.

* 1. El empresario garantizará de manera específica la protección de los trabajadores que, por sus características personales o estado biológico conocido, incluidos aquellos que tengan reconocida la situación de discapacidad física, psíquica o sensorial, sean especialmente sensibles a los riesgos derivados del trabajo. Con este fin, debe tener en cuenta estos aspectos en las evaluaciones de los riesgos y, según éstas, adoptará las medidas de prevención y de protección necesarias. Los trabajadores no serán empleados en aquellos puestos de trabajo en los que, debido a sus características personales o estado biológico o por su discapacidad física, psíquica o sensorial debidamente reconocida, puedan ellos, los demás trabajadores u otras personas relacionadas con la empresa exponerse a situaciones de peligro o, en general, cuando se encuentren manifiestamente en estados o situaciones transitorias que no respondan a las exigencias psicofísicas de los respectivos puestos de trabajo.

2. Del mismo modo, el empresario debe tener en cuenta en las evaluaciones los factores de riesgo que puedan incidir en la función de procreación de los trabajadores y las trabajadoras, en particular por la exposición a agentes físicos, químicos y biológicos que puedan ejercer efectos mutagénicos o de toxicidad para la procreación, tanto en los aspectos de la fertilidad como del desarrollo de la descendencia, con el fin de adoptar las medidas preventivas necesarias. 
En determinadas ocasiones y circunstancias, esta variabilidad es aceptable, por ejemplo cuando la evidencia científica no es concluyente o está relacionada con la limitación de recursos, la capacidad organizativa o las diferentes realidades sociales o culturales. Pero, a menudo, es necesario reducir esta variabilidad en la toma de decisiones médicas, que se quiere sean las más acertadas y éticas posibles. Y cuando la evidencia científica es débil, el consenso profesional, basado en el conocimiento y la experiencia previa, se convierte en una alternativa o un complemento necesarios.

En este sentido, proponemos un documento de orientación con unas recomendaciones genéricas, la aplicación de las cuales puede dar apoyo y ayuda a los profesionales de los SP en cuanto a la valoración colectiva y a la priorización, caso a caso, aplicando los criterios, los métodos, las guías y el procedimiento de la medicina del trabajo, en especial de la vigilancia de la salud.

\section{¿Cómo identificar y clasificar el riesgo laboral derivado del SARS-CoV-2?}

Un riesgo laboral, en circunstancias normales, es un riesgo determinado por las condiciones de trabajo y asociado a la tarea que desarrollan los trabajadores y debe estar evaluado por el SP. La evolución de la pandemia de SARS-CoV-2 añade, modifica o amplifica el riesgo de enfermar en el trabajo, por el riesgo de contacto con coronavirus SARS-CoV-2 circulantes con contagio directo (a través del contacto con personas con COVID-19, sean trabajadores, clientes o usuarios) o por contagio indirecto (a través del contacto con fómites de enfermos de COVID-19 o con superficies contaminadas con el virus), aparte de suponer muy frecuentemente la intensificación o modificación de los riesgos psicosociales.

Es por ello por lo que estamos ante un riesgo comunitario o poblacional de contacto en todos los entornos, y en el caso que nos implica, también en el entorno laboral.

El problema añadido es que el riesgo de contagio y enfermar en el trabajo a través de las vías de contagio se pueden dar en el marco de unas condiciones de trabajo habituales.

Por ello estamos ante un riesgo laboral y también ambiental con impacto en los centros de trabajo y, por tanto, susceptible de consideración y más aun teniendo en cuenta la presencia, en estos centros de trabajo, de personas más vulnerables frente a la enfermedad y de trabajadores especialmente sensibles a la exposición a riesgos biológicos según lo previsto en el RD 664/1997. 


\section{Tablas orientativas para valorar el riesgo laboral de exposición al SARS-CoV-2}

Partiendo de la aplicación de criterios epidemiológicos y de los de la normativa de $P R L^{*}$ se puede hacer una clasificación orientativa del riesgo de exposición al SARS-CoV-2 según los lugares de trabajo:

Tabla 1: Niveles de riesgo laboral por exposición al SARS-CoV-2

\begin{tabular}{ll}
\hline $\begin{array}{l}\text { A) Riesgo bajo (el riesgo general, } \\
\text { comunitario o poblacional): }\end{array}$ & $\begin{array}{l}\text { Trabajadores/as de todas las ocupaciones, a excepción de } \\
\text { los del grupo B) y C) }\end{array}$ \\
\hline B) Riesgo medio: & Trabajadores/as que atienden directamente al público, con \\
& posible contacto personal, o que trabajan en la limpieza, \\
& desinfección y/o recogida o manipulación de residuos \\
& contaminados o fómites de personas infectadas. \\
\hline C) Riesgo alto y muy alto & Trabajadores/as que trabajan en contacto directo en la \\
& atención, cuidado y transporte de personas enfermas \\
& (especialmente de personas que presentan patología \\
& respiratoria).
\end{tabular}

Fuente: Elaborada y adaptada a partir de las referencias bibliográficas

Esta clasificación evita voluntariamente especificar detalladamente puestos de trabajo lo que llevaría inevitablemente a olvidos y errores y pretende establecer unos criterios simples, aplicables por analogía y basados en la información mínima disponible en cada momento.

La evaluación del riesgo se formula en las condiciones basales del puesto de trabajo y sin la aplicación de medidas preventivas y, en concreto, del uso de equipos de protección individual (EPI). Todo ello sin perjuicio del necesario y preceptivo análisis de la viabilidad y eficacia de las medidas preventivas a la hora de emitir la indicación y la recomendación y muy especialmente en el caso de PEV y de TES.

\section{Criterios para orientar la toma de decisiones}

Más allá de la valoración de la evaluación de los riesgos biológicos de todos los puestos de trabajo, que ya desde antes de la pandemia tenía que estar disponible y era exigible a las empresas en aplicación del RD 664/1997 y de cara a actualizar los niveles de riesgo derivados del SARS-CoV-2, nos será útil clasificar los puestos

\footnotetext{
* El riesgo de contagio por el SARS_COV-2 en las empresas que mantienen la actividad productiva en el actual contexto de las regulaciones derivadas del estado de alarma no es ajeno, sino que forma parte de la emergencia de salud pública derivada de la pandemia de COVID-19.

Desde este punto de vista, hay que tener presente que en los casos en que el riesgo no deriva de la naturaleza de la actividad de las empresas y centros de trabajo (de las actividades comprendidas en el ámbito de aplicación del RD 664 / 1997 sobre la protección de los trabajadores contra los riesgos relacionados con la exposición a agentes biológicos durante el trabajo) no puede considerarse un riesgo laboral, sino que estaríamos ante un riesgo de que se produzca el contagio de una enfermedad infecciosa en el entorno laboral, es decir, que afecta a la salud pública en general.
} 
de trabajo en tres grupos, a partir de tres preguntas básicas que permiten decidir el nivel de riesgo del trabajo del caso en estudio:

Tabla 2: Preguntas para orientar la toma de decisiones

\begin{tabular}{|c|c|c|}
\hline & Pregunta & $\begin{array}{l}\text { Nivel de riesgo laboral en } \\
\text { caso de respuesta afirmativa }\end{array}$ \\
\hline $1 a$ & $\begin{array}{l}\text { ¿Trabajas con máquinas, información u otras actividades? } \\
\text { (trabajos no incluidos en los niveles B) y C) }\end{array}$ & A) \\
\hline $2 a$ & $\begin{array}{l}\text { ¿Trabajas con personas (no enfermas) o en la limpieza, la } \\
\text { desinfección y/o en la recogida o manipulación de residuos } \\
\text { contaminantes o fómites de persones enfermas } \\
\text { o infectadas? }\end{array}$ & B) \\
\hline $3 a$ & $\begin{array}{l}\text { ¿Trabajas con personas enfermas (especialmente } \\
\text { con enfermedades respiratorias)? }\end{array}$ & C) \\
\hline
\end{tabular}

Fuente: elaboración propia.

Esto implica que se concentre la mirada y la intervención donde realmente está concentrado el riesgo derivado de la naturaleza de la actividad laboral de forma prioritaria, esto es, en las actividades que prestan servicios de atención en entornos de asistencia médico-sanitaria, sociosanitaria y residencial (como servicios residenciales asistidos de personas mayores, discapacidad y salud mental) y de aquellas actividades que apoyan esta asistencia. En los demás puestos de trabajo, las estrategias preventivas, la determinación de especial sensibilidad, serán más residuales y se basarán a menudo más en el estudio de contactos, de casos positivos y de regreso al trabajo después de la curación tras un caso de la COVID-19.

Por otra parte, hay que considerar que, además del riesgo biológico en sí mismo, en ciertos entornos y especialmente en entornos de servicios básicos y esenciales, hay que tener presente una posible modificación o intensificación de los riesgos psicosociales (turnos, guardias, responsabilidad, sobrecargas, conflictos graves de rol, conflictos en las relaciones jerárquicas, contacto con la muerte y la enfermedad, cambios radicales de funciones por necesidades del servicio así como los asociados al teletrabajo o trabajo a distancia).

Por otra parte, en colaboración y coordinación con el sistema sanitario público, el SP deberá hacer una gestión eficaz y con celeridad de los trabajadores/as que inicien síntomas en el trabajo o de los que hayan tenido contactos en el entorno laboral con personas contagiadas o infectadas. En este sentido ante el estudio de contactos con personas contagiadas o infectadas, hay que tener presente estos escenarios de exposiciones de riesgo al coronavirus SARS-CoV-2 en el entorno laboral. 
Tabla 3: Escenarios de exposiciones de riesgo al coronavirus SARS-CoV-2 en el entorno laboral

\begin{tabular}{ll}
\hline Exposición de alto riesgo: & $\begin{array}{l}\text { Situaciones laborales en las que se puede producir un } \\
\text { contacto estrecho* con un caso de infección por el SARS- } \\
\text { CoV-2, sintomático. }\end{array}$ \\
\hline Exposición de bajo riesgo: & $\begin{array}{l}\text { Situaciones laborales en las que se puede producir un } \\
\text { contacte casual (que no sea un contacto estrecho) con un } \\
\text { caso de infección por el SARS-CoV-2. }\end{array}$ \\
\hline Baja probabilidad de exposición: & $\begin{array}{l}\text { Trabajadores/as que no tienen atención directa al público o, } \\
\text { si la tienen, se produce con más de dos metros de distancia, } \\
\text { o disponen de medidas de protección colectiva que evitan el } \\
\text { contacto (mampara de vidrio o metacrilato, etc.). }\end{array}$ \\
\hline
\end{tabular}

\footnotetext{
* Se clasifica como contrato estrecho:

- Cualquier persona que haya proporcionado cuidados a un caso probable o confirmado mientras el caso presentaba síntomas: trabajadores sanitarios que no han utilizado medidas de protección adecuadas, miembros familiares o personas que tengan otro tipo de contacto físico similar

- Convivientes, familiares o cualquier persona que haya estado en el mismo lugar que un caso probable o confirmado mientras el caso presentaba síntomas a una distancia < 2 metros durante un tiempo de, al menos, 15 minutos.

Fuente: Elaborada y adaptada a partir de las referencias bibliográficas.
}

\section{Concepto de especial vulnerabilidad de una persona y de especial sensibilidad de un trabajador/a.}

\section{Personas especialmente vulnerables (PEV): concepto y terminología}

Podríamos definir, en el contexto de este documento, como PEV a "aquellas personas que presentan factores de riesgo relacionados con su estado de salud que pueden hacer prever, con criterios epidemiológicos y clínicos, un incremento de la probabilidad de desarrollar la COVID-19 si se exponen a SARS-CoV-2 o de presentar una evolución clínica desfavorable en caso de padecer la COVID-19 ".

Las PEV, en cualquier caso, requieren por parte de la empresa y del SP una atención específica para adecuar la protección o las condiciones de trabajo más allá del nivel de compromiso social de la organización o del nivel de colaboración con el sistema de salud y de salud pública que haya establecido.

\section{Trabajadores/as especialmente sensibles (TES): concepto y terminología}

Los TES son aquellos "trabajadores/ as a los que la empresa garantizará de manera específica la protección de la salud debido a que, por sus características personales o estado biológico conocido, incluidos aquellos que tengan reconocida la situación de discapacidad física, psíquica o sensorial, sean especialmente sensibles a los riesgos derivados del trabajo. " 
Con este fin, se deben tener en cuenta estos aspectos en las evaluaciones de los riesgos y, en función de éstas, adoptar las medidas de prevención y de protección necesarias. Los TES no deben ser ocupados en aquellos puestos de trabajo donde puedan estar expuestos a situaciones de peligro o, en general, cuando se puedan encontrar manifiestamente en estados o situaciones transitorias que no respondan a las exigencias psicofísicas de los respectivos puestos de trabajo.

Porque queda claro, según el punto de vista epidemiológico inicial y clínico posterior, que ser considerada PEV en relación con la COVID-19 no supone automáticamente ser considerado TES de acuerdo con el artículo 25 de la LPRL. Por medio hay un elemento clave que es el "riesgo laboral" al que se tiene que exponer y que tendría que estar específicamente evaluado y del cual la empresa lo debe proteger en aplicación de los principios de la LPRL y del RD 664/1997 en concreto.

La iniciativa de valoración de una persona como PEV y/o como TES tendría que ser de la empresa a través del SP, pero también lo puede instar el propio trabajador/a.

\section{¿Cuáles son los grupos de patologías que pueden incluir personas especialmente vulnerables (PEV) a la COVID-19?}

Hay consenso en que hay ciertos grupos de patologías que concentran los casos de especial vulnerabilidad frente a la COVID-19, pero no hay una absoluta coincidencia en todos los diagnósticos posibles, en cuáles son estos grupos en concreto, y se entrelaza la vulnerabilidad de la frecuencia en infectarse o enfermar con el hecho de poder desarrollar procesos más graves ante la COVID-19. Pero más relevante es, sobre todo, que la vulnerabilidad supone tener más riesgo de gravedad por descompensación de las enfermedades de base o para desarrollar formas más graves de la COVID-19.

Determinar estos grupos puede orientar y ayudar a la detección de personas vulnerables a las cuales una valoración individualizada posterior por parte de los profesionales sanitarios del SP permitirá personalizar, por un lado, el riesgo al cual están expuestas y, del otro, su situación clínica y las medidas preventivas y recomendaciones a adoptar.

La Tabla 4 se ha elaborado analizando las principales recomendaciones y orientaciones de las autoridades y organismos sanitarios. En concreto se han analizado las tablas del Departament de Salut (DS), la del Ministerio de Sanidad, Consumo y Bienestar Social (MSCBS), la del European Centre for Disease Prevention and Control (ECDC), la del National Institute for Health and Care Excellence (NICE) y la de los Centers for Disease Control and Prevention (CDC).

Del conjunto de tablas se ha seleccionado las equivalencias para elaborar la Tabla 4 que pretende ser sencilla e inteligible, no exhaustiva ni cerrada con el fin de ayudar al médico/a al hacer la valoración de cada caso. 


\section{Listado de grupos de patologías y situaciones para orientar la determinación de PEV:}

Tabla 4: Grupos de vulnerabilidad personal

Edad > 65 años y > 50 años asociado a comorbilidad

Enfermedades cardíacas con insuficiencia cardíaca, cardiopatía isquémica activa e hipertensión arterial no controlada

Enfermedades respiratorias crónicas (EPOC, asma moderado/severa, etc.)

Enfermedades endocrinológicas graves o descompensadas (Diabetes mellitus, Enfermedad de Addison, Síndrome de Cushing, etc.) y obesidad mórbida (Índice de Masa Corporal > 40)

Neoplasias con tratamiento activo

Inmunodeficiencia e inmunosupresión (ver Tabla 5)

Enfermedad digestiva crónica grave

Enfermedades hepáticas crónicas graves

Enfermedad renal crónica con Insuficiencia renal grave (en tratamiento con diálisis)

Embarazo

Trastornos psiquiátricos graves (depresión mayor en fase de descompensación, crisis de panick attack, Ideación autolítica, dependencia a tóxicos, etc.)

Fuente: Elaborada y adaptada a partir de las referencias bibliográficas

Hay que decir que estas tablas y recomendaciones se refieren a las personas en general y no específicamente a las personas trabajadoras, grupo poblacional con unas características propias que sí se ha tenido en cuenta en la elaboración de la Tabla 4.

La Tabla 4, además, incluye los trastornos psiquiátricos graves que no aparecen en ninguna de las cinco fuentes analizadas y consultadas. Y no se han incluido por ser, como es el caso de los otros grupos de patologías, situaciones que supongan un mayor riesgo de contraer la COVID-19 o de hacer que su curso clínico pueda ser más grave en caso de contraerla, como es el criterio principalmente aplicado a las recomendaciones analizadas. La decisión de incluirlas se ha basado en cuatro razones primordiales:

1. El impacto que tienen estos trastornos mentales y psiquiátricos sobre la conducta que supone dificultades en la aplicación de medidas preventivas, higiénicas, organizativas y el uso adecuado de los EPI, etc., al tiempo que puede 
suponer mayor incumplimiento de los rituales y de los protocolos de prevención por un aumento de la angustia o falta de energía vital.

2. Pueden suponer una autopercepción alterada del estado de salud por consumo de fármacos que pueden mediatizar el dolor, la fiebre, el malestar general y que retrasen el diagnóstico.

3. Estos trastornos, por su descompensación, pueden impactar en negativo sobre los ciudadanos y afectar la seguridad de terceros en momentos de tensión máxima, y de trabajo bajo presión.

4. Se ha considerado, además, que estos trastornos psíquicos pueden favorecer la presentación de burnout y estrés postraumático por la exposición a niveles elevados de contaminación emocional.

La Tabla 4 no incluye procesos agudos que requieran atención y asistencia temporal especial o periodos de IT.

\section{Criterios para orientar la toma de decisiones}

Como actividad médica y procedimiento diagnóstico, la toma de decisiones debe basarse en el conocimiento de la evaluación de riesgos y de la situación clínica del trabajador/a de acuerdo con la historia clínica, la anamnesis, el conocimiento de las ausencias al trabajo por motivos de salud y los periodos de incapacidad temporal (IT) y si es necesario, las exploraciones, el tratamiento establecido de forma continuada, los informes disponibles y las consultas que se puedan realizar con los profesionales que lleven su proceso clínico. Se deberían aplicar criterios basados, siempre que sea posible, en la evidencia científica y la experiencia profesional, y nunca aplicar otros criterios basados en el saber convencional, opiniones o suposiciones sin fundamento científico o de carácter moral o de otro tipo.

Algunas de las condiciones de salud que habrá que tener en consideración, a la hora de evaluar el caso y la determinación, son las siguientes:

- Sexo

- Edad

- Motivo de la consulta, origen de la determinación y percepción de la propia salud.

- Duración, evolución y situación actual del proceso o procesos y grado de estabilidad clínica de los mismos.

- Antecedentes patológicos y de IT previos vinculadas al proceso i al motivo de la consulta.

- Histórico de recomendaciones derivadas de la vigilancia de la salud.

- Discapacidades, capacidades varias o invalideces permanentes previamente reconocidas. 
- Cualquier otra patología concomitante o característica personal que pueda influir.

- En caso de reincorporación de trabajadores/as curados de la COVID-19, atender especialmente siempre que sea posible, su nivel inmunitario específico para el SARS-CoV-2.

\section{¿Qué personas pueden ser consideradas trabajadores/as especialmente sensibles (TES) frente al riesgo laboral derivado del SARS- CoV-2?}

Cabe considerar como muy probable trabajador/a especialmente sensible (TES) a cualquier persona que:

- forme parte de los grupos de especial vulnerabilidad recogidos en la Tabla 4,

- ocupe un lugar de trabajo donde la evaluación de riesgos actualizada haya determinado un riesgo medio, alto o muy alto en relación con la exposición al coronavirus SARS-CoV-2,

- que les medidas preventivas adoptadas no puedan garantizar el control del riesgo y,

- que la valoración del médico/a del trabajo del SP así lo dictamine.

\section{Información adicional para situaciones determinadas para ciertas patologías o tratamientos}

Al aplicar los criterios genéricos de la tabla de grupo de patologías y situaciones orientativas para identificar PEV de inmunosupresión, y por analogía con las recomendaciones aceptadas de limitaciones de la administración de vacunas por agentes vivos o vivos atenuados, se recomienda la utilización de estos criterios por los que se puede considerar TES un trabajador/a con una inmunodeficiencia de alto nivel, y recomendar una valoración clínica especialmente rigurosa de los que se encuentran en un nivel bajo de inmunosupresión. 
Tabla 5: Inmunodeficiencias según el grado de inmunosupresión

\section{Inmunosupresión de alto nivel}

- Inmunodeficiencia primaria combinada de linfocitos B y T (por ejemplo, inmunodeficiencia combinada grave)

- Quimioterapia oncológica

- Infección por VIH y un recuento de linfocitos T-CD4 <200 células / $\mathrm{mm}^{3}$

- Recepción de agentes quimioterápicos (por ejemplo, Ciclofosfamida, metotrexato, micofenolato) y combinaciones de fármacos inmunosupresores para enfermedades hematológicas

- Terapia diaria de corticoides en una dosis de 20 mg de prednisona o equivalente, durante $\geq 14$ días

- Terapia con determinados inmunomoduladores biológicos, como los antagonistas del factor de necrosis tumoral alfa -TNF-a-(por ejemplo, adalimumab, certolizumab, infliximab, etanercept y golimumab), anticuerpos monoclonales antilinfócitos B (por ejemplo, rituximab) o anticuerpos monoclonales antilinfócitos T (por ejemplo, alemtuzumab).

- Durante los dos primeros meses posteriores al trasplante de un órgano sólido

- Dentro de los dos primeros meses posteriores a un trasplante de progenitores hematopoyéticos (TPH). Frecuentemente, las personas receptoras de TPH pueden tener alto grado de inmunosupresión durante un período mucho más prolongado, dependiendo del tipo de trasplante (el tiempo es superior en el caso de alotrasplantes), el tipo de donante y el origen de las células madre y las complicaciones posteriores al trasplante, como la enfermedad de injerto contra el huésped (MECH) y su tratamiento.

\section{Inmunosupresión de bajo nivel}

- Infección con VIH sin síntomas y un recuento de linfocitos T-CD4 2200 células/mm3

- Recepción durante $\geq 14$ días de una dosis diaria inferior de corticoides sistémicos la cual ocasiona inmunosupresión de alto nivel, o aquellos que reciben terapia de corticoides en días alternos

- Recepción de metotrexato en dosis de $\leq 0,4 \mathrm{mg} / \mathrm{kg} / \mathrm{semana}$, azatioprina en dosis de $\leq 3 \mathrm{mg} / \mathrm{kg} / \mathrm{día}$ o 6-mercaptopurina a dosis de $\leq 1,5 \mathrm{mg} / \mathrm{kg} / \mathrm{día}$

Fuente: Adaptación en el ámbito laboral de la 2013 IDSA clinical practice guideline for vaccination of the immunocompromised host 


\section{Situaciones especiales no vinculadas a enfermedades (aclaraciones):}

Trabajadoras embarazadas y lactantes:

Hay pocos datos referentes a la infección por SARS-CoV-2 durante el embarazo y la lactancia aunque se pueden inferir posibles efectos del coronavirus en este colectivo.

Por los datos disponibles, se considera poco probable que el embarazo o la lactancia supongan un incremento del riesgo de contagio ni un incremento de la gravedad clínica en caso de COVID-19. En cuanto a la transmisión vertical, tampoco hay evidencia (antes, durante o después del parto, por lactancia materna) del SARS-CoV-2 en mujeres que adquieren la infección durante el tercer trimestre de embarazo.

Por todo ello, y dado lo que supone en particular el embarazo y lo que tiene de singular, parece que hay que recomendar especial prudencia y aplicar el principio de precaución porque hay que considerar que la ausencia de evidencia de un riesgo no supone la evidencia de la ausencia del riesgo.

Para ello se propone aplicar también en estos casos, como en el de la inmunosupresión ya comentado anteriormente, los criterios de riesgo establecidos a la hora de administración, en general, de vacunas vivas y vivas atenuadas. Por eso:

Trabajadoras embarazadas:

Se sugiere aplicar en caso de personas embarazadas los criterios de TES y, por tanto, evitar cualquier riesgo laboral que no se pueda controlar con garantía de seguridad.

Trabajadoras lactantes:

Se sugiere no aplicar los criterios de TES y recomendar trabajar con las medidas que su nivel de riesgo y su situación de salud individual indiquen a criterio del médico/a del trabajo.

Trabajadores/as de más edad:

A pesar de figurar en algunas de las relaciones de personas con riesgo de desarrollar complicaciones en caso de COVID-19, con respecto a las personas de 60 a 65 años, no hay ninguna evidencia científica, más allá de la comorbilidad que pueda presentar cada persona, que justifique la aplicación de este criterio cronológico de forma sistemática.

Por ello se aconseja no incluirlos en los grupos de situaciones en las que aplicar de forma aislada los criterios de PEV ni TES y se recomienda incluirlos en el grupo de >50 a 65 años y, en función de la comorbilidad que puedan presentar, que el médico/a del trabajo decida las medidas que su nivel de exposición al riesgo y su situación de salud individual recomienden. 


\section{¿Qué actuaciones orientativas hay que llevar a cabo desde el SP ante una PEV y un TES derivado del SARS-CoV-2?}

\section{Personas especialmente vulnerables (PEV).}

- Incrementar, si es necesario, los niveles de protección general para todos los puestos de trabajo y por el conjunto de trabajadores/as dictados por la autoridad sanitaria, teniendo en cuenta que el coronavirus SARS-CoV-2 es un agente de la Tabla 2 del RD 664/1997, pero sin profilaxis ni tratamiento actualmente.

- Valorar otras medidas, en el contexto clínico, epidemiológico y social concreto de cada organización y de cada persona, posibles modificaciones de las condiciones de trabajo y funciones en las que hay que considerar el teletrabajo y el trabajo a distancia.

- Otras en función de cada contexto organizacional.

\section{Trabajadores/as especialmente sensibles (TES)}

En el caso de los considerados trabajadores/as especialmente sensibles (artículo 25 de la LRPL), la empresa debe tomar medidas específicas y especiales de acuerdo con su especial sensibilidad:

- Medidas preventivas, de todo tipo, para minimizar el riesgo de carácter técnico, organizativo, información y formación, EPI, etc., adecuadas al nivel de riesgo y reforzadas si es necesario, ya que, a pesar de ser el SARS-CoV-2 un agente de la Tabla 2 del RD 664/1997 no tiene ni profilaxis ni tratamiento actualmente.

- Cambio de puesto de trabajo o adaptación o cambio de las condiciones de trabajo que puede incluir restricciones concretas o el teletrabajo y el trabajo a distancia, entre otros.

- Cuando lo anterior no sea posible:

- Uso de permisos previstos en el convenio colectivo o en el contrato de trabajo.

- Tramitar la prestación de IT en coordinación con el sistema sanitario público.

- En el caso de embarazo, y de acuerdo con la gestante, tramitar la prestación de permisos de riesgo para embarazo en la Mutua Colaborada con la Seguridad Social (MCSS).

- Otras en función de cada contexto organizacional. 


\section{Modelo de informe médico}

\section{Informe de Vigilancia de la salud por especial sensibilidad al COVID-19}

Redactar primero la introducción que corresponda en el contexto de cada organización y la situación, en cada momento, de la pandemia por SASR-CoV-2, si procede, las referencias legales que corresponda.

Dado que: el/la señor/a forma parte de los servicios básicos y/o estratégicos de la empresa/organización XXXXXXX

Revisados: (la situación clínica, la evaluación de riesgos, etc.)

\section{Informo:}

a. Que XXXXXXXXXXXXXX es un trabajador/a especialmente sensible a los riesgos derivados de la exposición al SARS-CoV-2.

b. Que XXXXXXXXXXXXXXXXX por sus condiciones propias de salud es una persona especialmente vulnerable frente al riesgo de COVID-19.

c. Que XXXXXXXXX por sus condiciones propias de salud, NO es una persona especialmente vulnerable frente al riesgo de exposición al SARS-CoV-2 y por este motivo puede formar parte de los equipos de los servicios básicos o estratégicos y puede desempeñar su trabajo habitual, incluidas tareas presenciales.

Corresponde a la empresa adoptar las medidas pertinentes a la vista de este informe de acuerdo con las siguientes recomendaciones:

1.....

2. ....

3. ....

4. ....

Se aconseja aplicar estas recomendaciones a partir de la firma de este informe hasta la fecha: XXXXXXXX

Que firmo a los efectos oportunos

Atentamente,

El médico/a del trabajo 


\section{Siglas y acrónimos utilizados en este documento}

CDC: Centers for Disease Control and Prevention

DS: Departament de Salut

DTASF: Departament de Treball, Afers Socials i Famílies

ECDC: European Centre for Disease Prevention and Control

GTPSVTESSARS-CoV-2: Grupo de Trabajo para la elaboración de criterios para la gestión de personas especialmente vulnerables y trabajadores/as especialmente sensibles frente al SARS-CoV-2 en las empresas de Cataluña

IT: Incapacidad temporal

LRPL: Ley de prevención de riesgos laborales

MCSS: Mutua Colaboradora con la Seguridad Social

MSCBS: Ministerio de Sanidad, Consumo y Bienestar Social

MTES: Ministerio de Trabajo y Economía Social

NICE: National Institute for Health and Care Excellence

OMS: Organización Mundial de la Salud

PEV: Persona especialmente vulnerable

PRL: Prevención de riesgos laborales

RSP: Reglamento de los servicios de prevención

SCSL: Associació Catalana de Salut Laboral

SP: Servicio de Prevención

TES: Trabajador/a especialmente sensible 


\section{Bibliografía y enlaces de interés}

1. Ley de Prevención de Riesgos Laborales. L. N.o 31/1995 (8 Nov 1995).

2. Reglamento de los Servicios de Prevención. RD 39/1997 (17 En 1997).

3. Protección de los Trabajadores contra los Riesgos relacionados con la Exposición a Agentes Biológicos durante el Trabajo. RD 664/1997 (12 Mayo 1997).

4. Criterios Básicos sobre la Organización de Recursos para desarrollar la Actividad Sanitaria de los Servicios de Prevención. RD 843/2011 (17 Jun 2011).

5. Procediment d'actuació enfront de casos d'infecció pel nou coronavirus SARSCoV-2. Actualización de 4 de abril de 2020. Departament de Salut. Generalitat de Catalunya.

6. Informació cientificotècnica. Malaltia per coronavirus, COVID-19 Actualización de 4 de abril de 2020. Ministerio de Sanidad, Consumo i Bienestar Social.

7. COVID-19, Personas con factores de riesgo por enfermedades graves. Centers of Disease Control and Prevention.

8. Preguntas y respuestas COVID-19. European Centre for Disease Prevention and Control (ECDC).

9. Consejos para personas de alto riesgo (Coronavirus-Covid-19). National Institute for Health and Care Excellence (NICE).

10. Guia d'actuació i collaboració dels serveis de prevenció de riscos laborals per fer front a la pandèmia de COVID-19. Actualizado: 25.3.2020. Departament de Salut. Generalitat de Catalunya.

11. Procedimento de actuación para los servicios de prevención de riesgos laborales frente a la exposición al SARS-CoV-2. Actualitzado a 30 de marzo de 2020. Ministerio de Sanidad, Consumo y Bienestar Social.

12. Manual de vacunacions de Catalunya. Document de Salut. Generalitat de Catalunya.

13. Guia de práctica clínica IDSA 2013 para la vacunación del huésped immunocomprometido. 


\section{Composición del grupo de trabajo GTPSVTESSARS-CoV-2 y relación de revisores finales}

\section{Coordinador}

Jaume de Montserrat i Nonó

\section{Redactores}

Jaume de Montserrat i Nonó

Ramona Garcia Macià

Mari Cruz Rodriguez Jareño

\section{Grupo de Trabajo}

Ma Carme Bernad Castillo (médico del trabajo)

Antoni Carrillo Castillo (médico del trabajo)

Jaume de Montserrat i Nonó (médico del trabajo)

Ramona Garcia Macià (médica del trabajo)

Eduard Gaynés Palou (médico del trabajo)

Marta Gil Villares (médica del trabajo)

Josep Maria Molina Aragonés (médico del trabajo)

Miquel Mira Muñoz (médico del trabajo)

Pere Plana i Almuní (médico del trabajo)

Montse Puiggenè Vallverdú (médica del trabajo)

Elisabeth Purtí Pujals (médica del trabajo)

Mari Cruz Rodriguez Jareño (médica del trabajo)

\section{Revisores finales}

Sonia Alonso Mediavilla (enfermera del trabajo)

Cristina Abadia Castelló (médica del trabajo)

Leyre de la Peña Perea (médica del trabajo)

Joan Inglés Torruella (médico del trabajo)

Xavier Orpella Garcia (médico del trabajo)

Àngel Plans Cañamares (médico del trabajo)

José María Ramada Rodilla (médico del trabajo)

Eugenia Sánchez Flores (médica del trabajo) 